\title{
OC125, M11 and OV197 epitopes are not uniformly distributed in the tandem-repeat region of CA125 and require the entire SEA domain
}

\author{
Alessandro Bressan ${ }^{\mathrm{a}, *}$, Francesca Bozzo ${ }^{\mathrm{a}}$, Carlo Alberto Maggi ${ }^{\mathrm{b}}$ and Monica Binaschi ${ }^{\mathrm{a}}$ \\ ${ }^{a}$ Pharmacology Department, Menarini Ricerche S.p.A, Pomezia, Rome, Italy \\ ${ }^{\mathrm{b}}$ Menarini Ricerche S.p.A, Florence, Italy
}

\begin{abstract}
The human cancer antigen 125 (CA125) is over-expressed in epithelial ovarian cancer cells and it plays a role in the pathogenesis of ovarian cancer. This protein presents a repeat region containing up to sixty tandem repeat units. The anti-CA125 monoclonal antibodies have been previously classified into three groups: two major families, the OC125-like antibodies and M11-like antibodies, and a third group, the OV197-like antibodies. A model in which a single repeat unit contains all the epitopes for these antibodies has been also proposed, even if their exact position is still undetermined. In the present work, the affinities of the monoclonal antibodies, representative of the three families, have been investigated for different CA125-recombinant repeats through Western blot analysis. Different patterns of antibody recognition for the recombinant repeats show that CA125 epitopes are not uniformly distributed in the tandem repeat region of the protein. The minimal region for the recognition of these antibodies has been also individuated in the SEA domain through the subcloning of deleted sequences of the highly recognized repeat- 25 (R-25), their expression as recombinant fragments in E. coli and Western blot analysis. Obtained data have been further confirmed by ELISA using the entire R-25 as coating antigen.
\end{abstract}

Keywords: CA125, ovarian carcinoma, epitope type, SEA domain, monoclonal antibodies

\section{Introduction}

The human cancer antigen 125 (CA125) is a membrane-associated mucin-type glycoprotein [16,17], encoded by the MUC16 gene. It was identified through the OC125 monoclonal antibody (mAb) that bound to the surface of the ovarian cancer cells OVCA 433 [27]. It is normally found in abdominal fluid and in cervical mucus $[10,15]$, in female reproductive organs, in pleura, pericardium and peritoneum, in the epithelium of the ocular surface and the in the upper respiratory tract $[1,11,23,38]$.

*Corresponding author: Alessandro Bressan, Pharmacology Department, Menarini Ricerche S.p.A, Via Tito Speri 10, 00040, Pomezia, Rome, Italy. Tel.: +39 0691184510; Fax: +39 069100220; E-mail: ABressan@menarini-ricerche.it.
A remarkable aspect of CA125 is its over-expression in epithelial ovarian cancer cells as well as in other carcinomas $[6,9,18]$. The CA125 aberrant expression might play a role in the pathogenesis of ovarian cancer. It has been suggested that CA125 might protect the ovarian cancer cells from NK immune surveillance [22] and from the attack of complement factors [36]. Since CA125 interacts with mesothelin, a protein expressed by the mesothelial cells of peritoneum, it might promote the metastatic dissemination of ovarian cancer cells [13]. Besides, CA125 directly regulated cell growth, tumorigenesis and metastasis of ovarian cancer cells in in vitro experiments [5,29].

CA125 is released from the surface of the cells into the blood and it is used as ovarian tumoral marker in serum assays [25]. Although the CA125 detection 
shows low specificity and high rates of false positives in early disease, it represents a useful tool to monitor the progression or the regression of ovarian carcinomas after surgery and cytotoxic therapy [25,26].

The antigenic properties of CA125 are also considered for therapeutic approaches against ovarian cancer. Indeed, anti-CA125 mAbs were evaluated as radionuclide or cytotoxin conjugated [20,37], as anti-idiotype antibody in order to mime the OC125 epitope and to elicit an antitumor immune response [12,19], or as immunoglobulins to induce an antibody-dependent cellular cytotoxicity [3].

Many CA125 features may be elucidated by its molecular structure. The cloning of the human MUC16 gene provided some indication on the structure of the encoded protein $[33,34]$. It is up to 22,152 amino acid (aa) long, with a molecular weight of $2.5 \mathrm{MDa}$ that has to be doubled considering glycosilation. The protein consists of a highly glycosilated N-terminal extracellular region, a multiple repeat domain, containing up to sixty tandem repeat units, a carboxy terminal domain, presenting a potential proteolytic cleavage site proximal to the membrane, a transmembrane anchor and a short cytoplasmic tail [34]. It should be considered that CA125 may show biochemical and molecular heterogeneity depending on the biological sources, the use of different isolation techniques and different isoforms [8]. A single repeat unit is about 156 aa long [4, 34] and contains a SEA module, a module found in sea urchin sperm protein, enterokinase and agrin [24], which presents a sandwich three-dimensional structure formed by two alpha helices, four antiparallel beta strands and a hydrophobic core [24,32]. Several hypotheses for the SEA module function were proposed, like protein recognition, nucleic acid as well as sugar chain binding activity, interaction with matrix molecules [24,32].

All the antigenic domains of CA125 have been reported in the repeat region [14]. Indeed, twenty-six anti-CA125 mAbs defined only three antigenic domains, that were classified into three families [14]: two major groups, the OC125-like antibodies (group A) and the M11-like antibodies (group B), and a third group C represented by the OV197 antibody. At present, the exact position of CA125 epitopes in the repeat units remains undefined even if a model in which a single repeat unit encompasses all the three antigenic domains has been proposed [7,34].

The heterologous expression of one of the CA125 repeats, the repeat 11 (R-11: the numbering of the repeats starts from the $\mathrm{N}$-terminus of CA125 according to O'Brien et al. [34]), highlighted that the OC125 $\mathrm{mAb}$ poorly reacted with this unit both in antigen capture and cross-inhibition methods and that the prior binding of some mAbs from the group B enhanced subsequent OC125 recognition. It has been argued that CA125 epitopes need to be contained in the CA125 tandem repeat region to be optimally displayed and that they may be conformationally dependent $[7,14]$.

Although the amino acid sequences of CA125 repeats are highly homologous, they are not perfectly conserved $[33,34]$. We investigated the affinity of mAbs, representative of the CA125 antigenic domains, for several CA125 recombinant repeats in addition to R-11.

For the first time, the cloning and the heterologous expression of different repeat units of CA125, including the R-11, is here reported. The OC125 and the OV197 mAbs recognized the recombinant repeats with very different efficiencies. The M11 mAb recognized all the recombinant repeats, although with some differences. For high bindings to the recombinant repeats, incubations with single antibodies were sufficient, both in immunoblot and in ELISA analysis. A deletion analysis showed that the region of the R-25 containing the entire SEA domain was necessary to be recognized by CA125 mAbs.

These data indicate that the affinity of mAbs for the CA125 repeats dramatically depends on the primary structures of their amino acid sequences and confirm the conformational type of CA125 epitopes.

\section{Materials and methods}

\subsection{Materials}

Human ovarian tumor epithelial OAW28 cells were from ECACC, Salisbury, UK. They were routinely cultured in DMEM complemented with $10 \%$ FBS, 2 mM Glutamine, 20 IU/I Bovine Insulin and $1 \mathrm{mM}$ Sodium Pyruvate (Invitrogen, Paisley, UK).

For the cloning of the CA125-repeat units, amplification primers were synthesized by Primm (Milan, Italy). The DNA polymerase enzyme used was the PfuUltra ${ }^{\circledR}$ Hotstart DNA polymerase from Stratagene (La Jolla, CA, USA). The restriction enzymes were purchased from Roche Applied Science (Mannheim, Germany). The competent cells used for bacterial transformations derived from the JM109 E. coli strain and were purchased from Stratagene.

Western blots were performed with the anti-CA125 mouse mAb (OC125) (Calbiochem Merck, Darmstadt, 
Germany), the monoclonal mouse anti-human CA125 clone M11 (DakoCytomation, Carpinteria, CA, USA), the OV197 (Fujirebio Diagnostic, Göterborg, Sweden), the secondary antibody goat anti-mouse $\operatorname{IgG}(\mathrm{H}+\mathrm{L})$ HRP Conjugate (Bio-Rad, Hercules, CA, USA) and with the penta-His HRP conjugate antibody (Qiagen, Valencia, CA, USA).

ELISA on the purified His-tagged hCA125-repeat units were performed using Ni-NTA HisSorb 96-well plates from Qiagen, the mentioned primary antibodies and the peroxidase-conjugated affiniPure goat AntiMouse IgG, Fc $\gamma$ Fragment Specific as secondary antibody (Jackson Immunoresearch, West Grove, PA, USA). For the ELISA of the His-tagged murine DHFR, the primary antibody was a rabbit polyclonal antiDHFR antibody (Santa Cruz Biotechnology Inc., Santa Cruz, CA, USA), while the secondary antibody was the peroxidase-conjugated affinipure goat anti-rabbit IgG, Fc fragment specific from Jackson Immunoresearch.

\subsection{Methods}

\subsubsection{Cloning of His-tagged hCA125-repeat units in E. coli}

The cloning of CA125-repeat units was executed with QIAexpress Type IV Kit (Qiagen). This kit contains the expression vector pQE-30, which is used for the expression of $6 \times$ His-tagged recombinant proteins, and the expression vector $\mathrm{pQE}-40$ that expresses a His-tagged murine DHFR protein and is used as control or for chimeric constructs. His-tagged proteins may be purified through nickel-nitrilotriacetic acid (Ni-NTA) metal affinity chromatography and used as antigen in ELISA with Ni-NTA coated plates, according to the instructions of the kit manual.

Total RNA was isolated and purified from OAW28 cells using the SV Total RNA Isolation System from Promega (Madison, WI, USA); RNA yield and the integrity were determined spectrophotometrically and by gel electrophoresis respectively. The cDNA was synthesized with the Reverse Transcription System (Promega) and used in PCR for the amplification of CA125 tandem repeat units. For the cloning of the R11 unit, R11-U and R11-L primers were synthesized, according to the oligo sequences published by $\mathrm{T} \mathrm{J}$ O'Brien et al. [34].

A second repeat cloning was performed with the forward primer U-I and the reverse primer L-X (Table 1). PCR reaction mixtures were set up with cDNA deriving from $300 \mathrm{ng}$ of total RNA, 25 pmol of each primer, $50 \mathrm{nmol} \mathrm{MgCl}_{2}, 50 \mathrm{nmol} \mathrm{dNTP}$, buffer $1 \times$ and
$2.5 \mathrm{U}$ Pfu, in a final volume of $50 \mu \mathrm{l}$. The PCR amplification protocol for R-11 was $5 \mathrm{~min}$ at $95^{\circ} \mathrm{C}$, for the initial denaturation and enzyme activation, $7 \mathrm{cy}-$ cles consisting of denaturation at $95^{\circ} \mathrm{C}$ for $1 \mathrm{~min}$, annealing at $61^{\circ} \mathrm{C}$ for $1 \mathrm{~min}$ and extension at $72^{\circ} \mathrm{C}$ for $2 \mathrm{~min}, 25$ cycles at $95^{\circ} \mathrm{C}$ for $1 \mathrm{~min}$ and $72^{\circ} \mathrm{C}$ for $3 \mathrm{~min}$, and a final step at $72^{\circ} \mathrm{C}$ for $7 \mathrm{~min}$. The amplification with the second primer pair was performed at an initial annealing of $63^{\circ} \mathrm{C}$, instead of the calculated $65^{\circ} \mathrm{C}$, to amplify more different repeat units in sub-stringent conditions. Amplifications took place in a 7300 Real Time PCR system (Applied Biosystems, Foster City, CA, USA). The amplified products were checked by gel electrophoresis and purified with the PCR cleanup Gel extraction kit (Macherey-Nagel, Duren, Germany). Both the expression vector $\mathrm{pQE}-30$ and the purified inserts were digested with Bam $\mathrm{HI}$ and Hind III restriction enzymes. The digestion products were separated by electrophoresis. The DNA band corresponding to the digested vector was excised and purified with the described kit, while the digested inserts were run and excised from the low melting gel NuSieve GTG (Lonza, Walkersville, MD, USA), and directly used as melted gel in the ligase reaction. The reaction of ligation between the expression vector and insert was performed with the Rapid DNA ligation kit (Roche Applied Science). Ligation mixtures were used to transform JM109 E. coli competent cells. Bacterial clones were grown and plasmidic DNA was prepared with Nucleobond Xtra Maxi Plus and Nucleospin Plasmid kits from Macherey-Nagel. Plasmidic DNAs were characterized by restriction analysis.

\subsubsection{Subcloning of His-tagged deleted regions of the $R-25$ unit in E. coli}

The subcloning of nucleotidic sequences encoding R-25 subregions, both in pQE-30 and in pQE-40 expression vectors, was performed as described above for the cloning of the entire repeat in pQE-30. Ten nanograms of $\mathrm{pQE} 30-\mathrm{R} 25$, the vector containing the entire cloned R-25 sequence, were used as template in PCR reactions. Amplification primers, besides the U-I and L-X primers described as above, were: the upper primers U-II, U-III, the lower primers L-VIII and LIX (Table 1). Schematic representations of all the products encoded by the obtained clones are reported in Fig. 3A, for the pQE-30 subclones, and in Fig. 4A, for the chimeric products with murine DHFR.

\subsubsection{Sequencing}

The nucleotidic sequences of inserts, as well as the correct in frame ligation, were determined through nucleotidic sequencing by Primm (Milan, Italy). 
Table 1

Primers used for the amplification and cloning of CA125 repeats and the R-25 regions

\begin{tabular}{lll}
\hline Primer name: & Primer sequence (5'-3') & Amplified CA125 regions \\
\hline R11-U: & ACCGGATCCGGCCACACAGAGCCTGGCCC & R-11 \\
R11-L: & TGTAAGCTTAGGCAGGGAGGATGGAGTCC & \\
U-I: & ACCGGATCCAGCCCCACAACTGCTGGCCCT & R-2, -7, -9, -25, -51 \\
L-X: & TGTAAGCTTGGGGAGGGAGGATGGAGTCCCTGA & \\
U-II: & CGGGGATCCGGCTCCAGGAAGTTCAACACCACA & R-25 regions \\
U-III: & CGGGGATCCGGTCCCATGTTCAAGAACACCAGT & \\
L-VIII: & CCGAAGCTTGTATAGCTGCTCCCTGTCCACT & \\
L-IX: & CGGAAGCTTGGTGAAACCATTGACATAGAGACT & \\
\hline
\end{tabular}

Restriction sites are underlined. GGATCC: Bam HI, AAGCTT: Hind III. U: upper primer, L: lower primer.

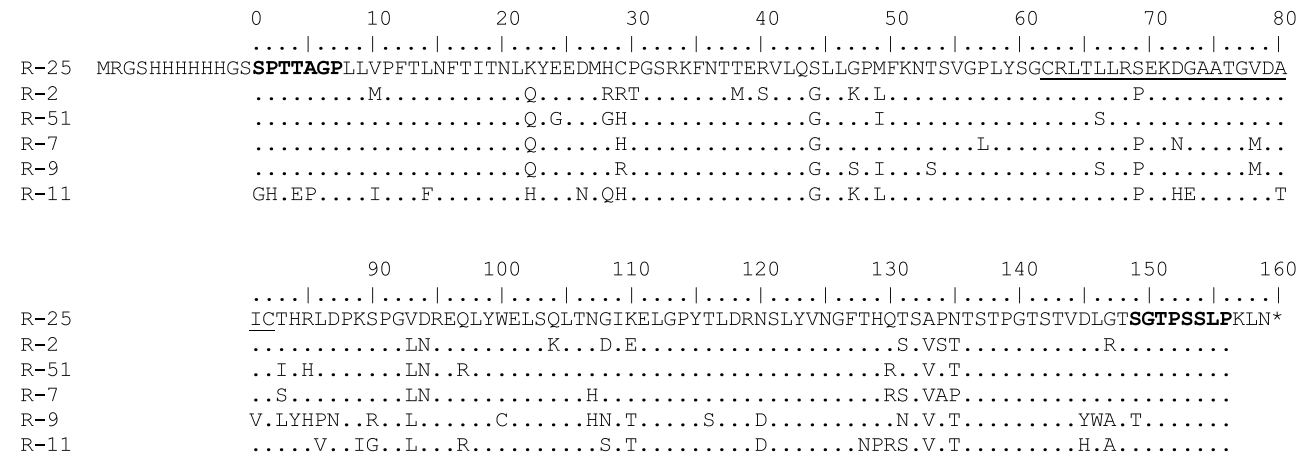

Fig. 1. Alignment of the amino acid sequences of six recombinant CA125 repeats subcloned and expressed in E. coli. Not aligned residues at the amino- and carboxy- ends are encoded by plasmidic sequences. The SEA domain extends from position 0 to 126 [32], while the cysteine loop region is underlined. Residues in bold are shared by the R-25, -2, -51, -7 and -9 , since they all derived from the primers used for the amplification of the R-25 encoding sequence (see Table 1).

\subsubsection{Expression of $6 \times$ His-tagged hCA125-repeat units in E. coli and Western blot analysis}

According to the QIAexpressionist handbook (Qiagen), the obtained bacterial clones were grown at $37^{\circ} \mathrm{C}$ to $\mathrm{OD}_{600}=0.5$ and induced for $4 \mathrm{~h}$ with $1 \mathrm{mM}$ IPTG to produce the $6 \times$ His-tagged hCA125repeat units. Bacterial pellets were lysed with Buffer B (100 mM NaH${ }_{2} \mathrm{PO}_{4}, 10 \mathrm{mM}$ Tris $\mathrm{Cl}, 8 \mathrm{mM}$ Urea, $\mathrm{pH}$ 8). NuPAGE ${ }^{\circledR}$ LDS Sample buffer with reducing agent was added to lysates that were run on NuPAGE ${ }^{\circledR}$ 4$12 \%$ Bis-Tris gel (Invitrogen, Grand Island, NY, USA) at $200 \mathrm{~V}$ for $30 \mathrm{~min}$. Proteins were electroblotted at $30 \mathrm{~V} 1 \mathrm{~h}$ on $0.2 \mu \mathrm{m}$ PVDF membrane (Bio-Rad) and the transfer was controlled with Red-Ponceau staining (Sigma, St. Louis, MO, USA). Membranes were incubated $1 \mathrm{~h}$ at room temperature (RT) in blocking buffer (5\% non-fat milk, $0.05 \%$ Tween-20, $1 \times$ PBS or in casein $1 \%$ ). The primary antibodies were incubated in the same buffer and at RT for $2 \mathrm{~h}$. Depending on the immuno-detection, the primary antibodies were $2.5 \mu \mathrm{g} / \mathrm{ml}$ anti-CA125 mouse mAb (OC125) or $2.5 \mu \mathrm{g} / \mathrm{ml}$ monoclonal mouse anti-human CA125 clone M11 or $2.5 \mu \mathrm{g} / \mathrm{ml}$ OV197 mAb. After three washes with wash buffer $(0.1 \%$ Tween-20, $1 \times$ PBS $)$ at RT for $10 \mathrm{~min}$, membrane were incubated with 1:3000 goat anti-mouse IgG $(\mathrm{H}+\mathrm{L})$-HRP conjugate at RT for $1 \mathrm{~h}$. After four washes, chemiluminescent detection was performed with the ECL Western Blotting Detection Reagents on X-ray film (Amersham GE Healthcare, Little Chalfont, UK). When $0.1 \mu \mathrm{g} / \mathrm{ml}$ Penta-His Antibody (Qiagen) was used as primary antibody, the blocking and incubation buffer was $1 \times$ PBS/Casein blocker (Bio-Rad). For reprobing with different antibodies, membranes were before incubated in stripping buffer (20 ml SDS 10\%, $12.5 \mathrm{ml}$ Tris $\mathrm{HCl} \mathrm{pH}$ $6.8,0.5 \mathrm{M}, 0.8 \mathrm{ml}$ betamercaptoethanol) at $50^{\circ} \mathrm{C}$ for $45 \mathrm{~min}$.

\subsection{Expression and purification of the $R-25$}

Protein expression and purification was performed following the handbook of the QIAexpress Type IV Kit (Qiagen). Briefly, both a selected bacterial clone expressing hCA125-repeat unit and the one expressing murine DHFR as control protein (Qiagen) were grown at $\mathrm{OD}_{600}=0.5$ and induced for $5 \mathrm{~h}$ with $1 \mathrm{mM}$ IPTG. Each bacterial pellet, derived from $0.5 \mathrm{~L}$ of culture, 


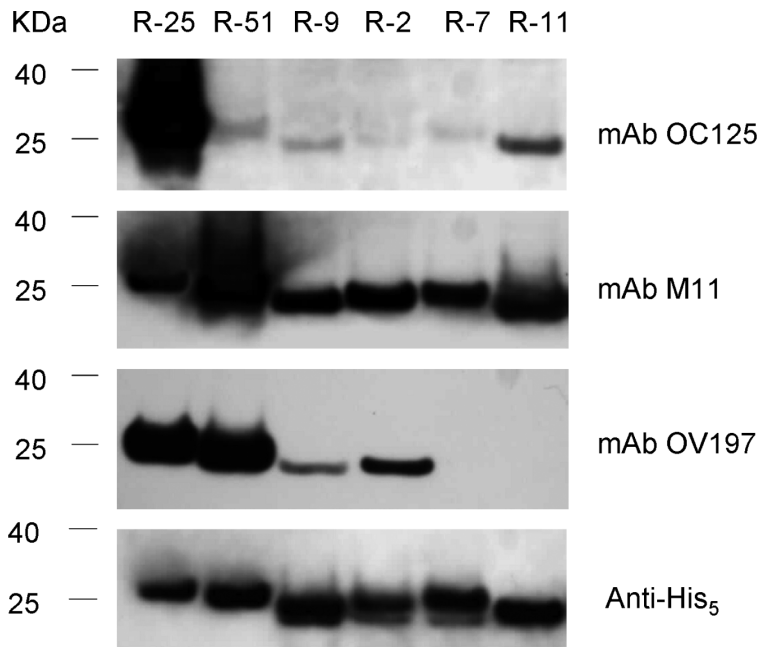

Fig. 2. Western blot analysis of six recombinant CA125 repeats. E. coli extracts containing the indicated recombinant repeats were probed with the monoclonal antibodies OC125, M11, OV197 and with the anti-penta histidine as control antibody. The numbering of the repeats starts from the N-terminus of CA125 according to O'Brien et al. [34].

was lysed through incubation with $100 \mathrm{ml}$ buffer B (see above) at RT for $1 \mathrm{~h}$. After $10000 \times \mathrm{g}$ centrifugation at RT for $30 \mathrm{~min}$, each supernatant was incubated with 1$1.5 \mathrm{ml}$ of $50 \% \mathrm{Ni}-\mathrm{NTA}$ resin (Qiagen) at RT for $1 \mathrm{~h}$, on a rotary shaker at $200 \mathrm{rpm}$. The supernatant-resin mixtures were loaded into empty columns. The columns were washed 3-4 times with $5 \mathrm{ml}$ buffer B, 5-7 times with $5 \mathrm{ml}$ buffer $\mathrm{C}\left(100 \mathrm{mM} \mathrm{NaH} \mathrm{PO}_{4}, 10 \mathrm{mM}\right.$ Tris $\mathrm{Cl}, 8 \mathrm{mM}$ Urea, $\mathrm{pH} 6.3$ ), and proteins were eluted 4-8 times with $0.5 \mathrm{ml}$ buffer $\mathrm{D}$ for protein monomers (100 $\mathrm{mM} \mathrm{NaH}{ }_{2} \mathrm{PO}_{4}, 10 \mathrm{mM}$ Tris $\mathrm{Cl}, 8 \mathrm{mM}$ Urea, $\mathrm{pH}$ 5.9) followed by 4-8 times with $0.5 \mathrm{ml}$ buffer $\mathrm{E}$ for protein multimers (100 mM NaH${ }_{2} \mathrm{PO}_{4}, 10 \mathrm{mM}$ Tris $\mathrm{Cl}, 8 \mathrm{mM}$ Urea, $\mathrm{pH} 4.5$ ). Eluted proteins were controlled on 10$20 \%$ SDS PAGE gels stained with Bio-Safe Coomassie G-250 (Bio-Rad). The elution fractions containing the most of the proteins were pooled and the resulting protein concentration was assessed with the Protein Assay of Bio-Rad.

\subsubsection{Immunodetection of the hCA125 R25 by ELISA}

The nickel-nitrilotriacetic acid (Ni-NTA) is also used in the Ni-NTA HisSorb 96-well plates to capture tagged protein from cleared lysates as well as to perform ELISA. ELISA on the purified His-tagged hCA125-repeat unit and His-tagged murine DHFR control were performed following the instruction of the QIAexpress Detection and Assay Handbook (Qiagen) with some modifications. Briefly, for the coating of the plates, $250 \mathrm{ng}$ of $6 \times$ His-hCA125-repeat unit, in $200 \mu \mathrm{l}$ of blocking buffer $(0.2 \%$ BSA, PBS $1 \times, \mathrm{pH}$ 7.4), were added to each well and incubated at RT for $1 \mathrm{~h}$. After four washes with $300 \mu \mathrm{l}$ PBS-Tween $(0.05 \%$ Tween-20 in PBS $1 \times$ ), $200 \mu \mathrm{l} /$ well of $1 \mu \mathrm{g} / \mathrm{ml} \mathrm{mAbs}$ in blocking buffer were added and incubated at RT for 2 hours. After four washes with $300 \mu$ l PBS-Tween, $200 \mu \mathrm{l} /$ well of secondary antibody in blocking buffer (1:20000 Peroxidase-conjugated AffiniPure Goat AntiMouse IgG, Fc $\gamma$ Fragment Specific) were added and incubated at RT for 1 hour. After four washes with $300 \mu \mathrm{l}$ PBS-Tween, $200 \mu \mathrm{l} /$ well ABTS solution (Roche Applied Science) was added and incubated until color development. The detection at $\mathrm{OD}_{405}$ was performed with the Infinite M200 counter (Tecan, Salzburg, Austria). For the ELISA of the His-tagged murine DHFR, more stringent conditions were used to reduce the background signal. The blocking buffer was $1 \%$ BSA, PBS $1 \times, \mathrm{pH} 7.4$, and the dilution of the secondary antibody, the peroxidase-conjugated goat anti-rabbit IgG, was 1:20000.

\section{Results}

\subsection{Cloning of six different CA125-repeat units}

The cloning of several repeat units from the tandemrepeat domain of CA125 was performed through the reverse-transcription and the PCR amplification of total RNA from OAW28, an ovarian cancer cell line expressing the glycoprotein [2]. Since a possible immunogenic region, the "cysteine loop", has been previously recognized within the R-11 by O'Brien et al. [34], the encoding sequence for the R-25, expressing one of the most represented C-C region of CA125, was isolated by amplification with specific primers (Table 1, Fig. 1). Anyway, the PCR was executed in sub-stringent conditions for the annealing temperature, thereby isolating not only the encoding sequence for the R-25 but also the sequences for R-2, -7, -9 , and -51 . The amino acid sequences deduced from the nucleotidic sequences of other isolated $E$. coli clones were redundant compared to the above-mentioned, indicating that all the possible repeats amplified with this primer pair were probably isolated. The R-11 encoding sequence was amplified with a specific primer pair and cloned to confirm literature data [34]. As expected and reported in Fig. 1, the alignment of the amino acid sequences, deduced from the nucleotidic sequences from bacterial clones, shows that all the repeats present the 

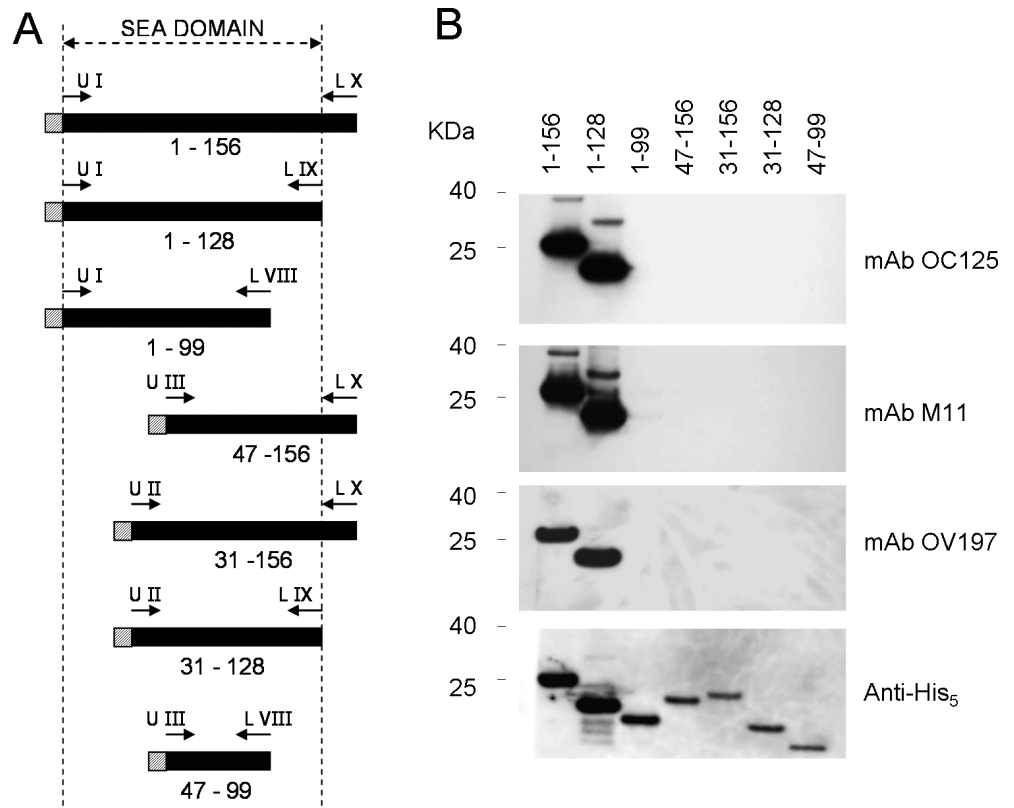

Fig. 3. Western blot analysis of recombinant regions of R-25. A: Diagram of the tested recombinant R-25 regions. Poly-histidine tags are indicated with hatched boxes. The primers used for the amplification of the encoding sequences are indicated and their sequences reported in Table 1 . The numbering of the recombinant regions corresponds to the amino acid positions into the repeat. B: E. coli extracts containing the recombinant regions were probed with the OC125, M11, OV197 mAbs and with the anti-penta histidine as control antibody.

SEA domain [32], the "cysteine-loop" region [34], as well as amino acid substitutions among them, indicating that the corresponding conformational structures could present some differences and suggesting further investigations on the recombinant repeats.

\subsection{Western blot analysis of the recombinant-repeat units}

The recombinant repeats were expressed in E. coli clones and bacterial extracts were analyzed by Western blot analysis. The OC125, M11 and OV197 mAbs, representative of the three principal groups of CA125 antibodies [14], bound to the recombinant repeats with very different efficiencies. Unexpectedly, OC125 mAb bound very strongly to the R-25 (Fig. 2) and, as already reported in published data [34], more weakly with the R-11. Faint bands were observed for the remaining repeats. M11 mAb recognized all the repeats, with the strongest signals for R-51. Also the OV197 mAb did not recognize the recombinant CA125 repeats homogeneously: it strongly bound R-25 and R-51 and, to a lesser extent, R-2 and R-9. The OV197 mAb did not recognize R-7 and R-11.

The amino acid differences in the repeat sequences could have also determined the observed slight mobility shifts during the electrophoretic run, as discussed below.

\subsection{Subcloning of $R-25$ deleted regions}

In order to characterize the minimal region of the R-25 able to interact with CA125 antibodies, a deletion analysis was performed by subcloning partial nucleotidic sequences of this repeat and by expressing the derived recombinant fragments in E. coli. As reported in Fig. 3A, progressive deletions were performed from both 5'- and 3'-nucleotidic ends. The lengths of the overlapping regions among the fragments were sufficient to maintain putative linear epitopes [35]. The above-mentioned deleted sequences were also subcloned in frame with the murine DHFR sequence (Fig. 4A), using the pQE40 vector, to express chimeric recombinant proteins. This second subcloning was performed to better uniform the expression of the R-25 fragments, obtaining more similar protein quantities in bacterial extracts, and to analyze antibody interactions with an independent conformational arrangement.

\subsection{Deletion analysis of R25 unit by Western blot}

The deleted R-25s expressed by the bacterial clones were analyzed by Western blot. As shown in Fig. 3B, only the fragments containing the entire SEA domain [32] was recognized by OC125, M11 and OV197 


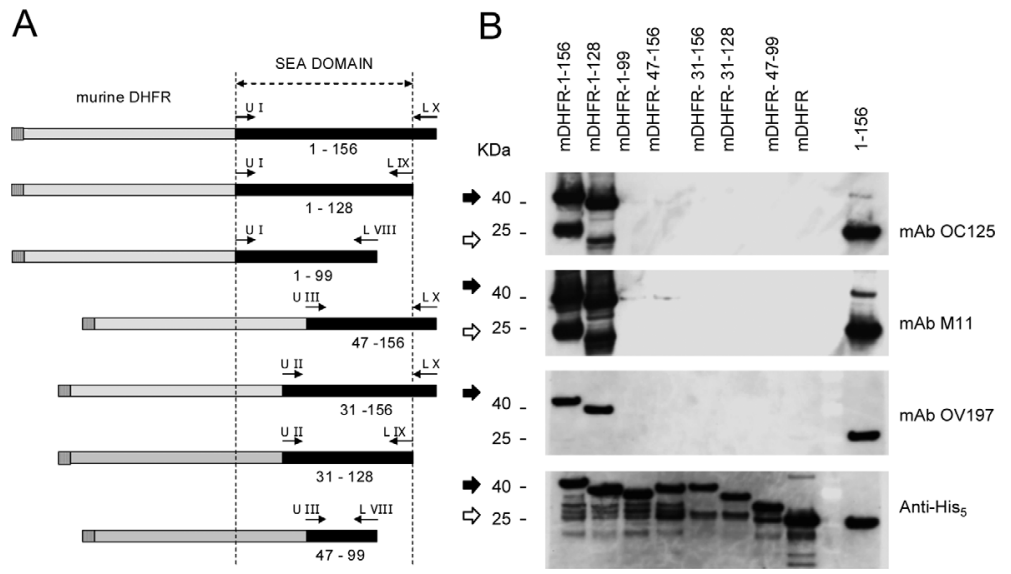

Fig. 4. Western blot analysis of chimeric regions of R-25. A: Diagram of the chimeric R-25 regions. The encoding sequences for the recombinant regions of the R-25, reported in Fig. 3, were subcloned in frame with the sequence encoding the murine DHFR. Poly-histidine tags are indicated with hatched boxes. The primers used for the amplification of the encoding sequences are indicated and their sequences are reported in Table 1 . The numbering of the recombinant regions corresponds to the amino acid positions into the repeat. B: E. coli extracts were probed with the OC125, M11, OV197 mAbs and with the anti-penta histidine as control antibody. Lane 1-156 was loaded with the not-chimeric R-25. The black arrows indicate the chimeric constructs, while the open arrows indicate degradation products.

mAbs. Shorter fragments did not interact with antibodies at all. In spite of the same experimental conditions used for the induction of protein expression in the bacterial clones, the detection of the R-25 fragments with the anti-pentaHis antibody, that recognize the common structure of a NH-terminal poly-histidine tag, revealed that they were produced in different quantities, probably due to the different transcriptional and/or translational efficiencies of the subcloned sequences as well as to different product stabilities in the bacterial environment.

The chimeric murine-DHFR/R-25 fragments were more uniformly expressed (Fig. 4B) but, once more, only those containing the SEA domain were recognized by the CA125 antibodies. Observed bands with lower molecular weights than expected were due to bacterial proteolysis. Noteworthy, only the proteolysed fragments with the same molecular weight of the R-25 and the SEA domain were recognized by OC125 and M11 antibodies, confirming that the integrity of this domain was necessary.

\section{5. $R 25$ recognition by CA125 mAbs through ELISA}

The amino-terminal poly-histidine tag of the recombinant R-25 was used to produce and purify the repeat through nickel-nitrilotriacetic acid (Ni-NTA) resin and to analyze its interactions with the anti-CA125 mAbs by ELISA using Ni-NTA coated plates. As reported in Fig. 5, also in ELISA the OC125, the M11 and the OV197 antibodies specifically bound to the repeat, thereby confirming the Western blot data with an independent immunological method.

\section{Discussion}

As previously reported, the $\mathrm{OC} 125 \mathrm{mAb}$, belonging to the group A of anti-CA125 antibodies, according to the ISOBM TD-1 classification [14], had shown little reactivity to the recombinant R-11 in immunoblot [34] and in antigen-capture assay [7], while, in the same experiments, the M11 mAb, belonging to the group $B$, reacted with high binding. Surprisingly, the OC125 $\mathrm{mAb}$ increased its affinity for the R-11 in the presence of competitor antibodies or after a pre-incubation with group B antibodies, indicating a conformational modulation of the structure of its epitope [7]. It has been suggested that the binding data obtained with the recombinant R-11 could be representative of CA125 interactions with antibodies and that their binding efficiency could depend on the conformational context of the repeats [7].

In this paper the subcloning and the expression in $E$. coli of several repeats, including the R-11, is reported. The R-25 was efficiently recognized not only by M11, but also by OC125, and OV197 mAbs in ELISA as well as in immunoblot assays, without any other concomitant antibody interaction. The OC125 mAb was less reactive with the R-11, as expected, and showed very low binding, if any, with the other repeats. The M11 mAb was generally reactive to all the repeats with 

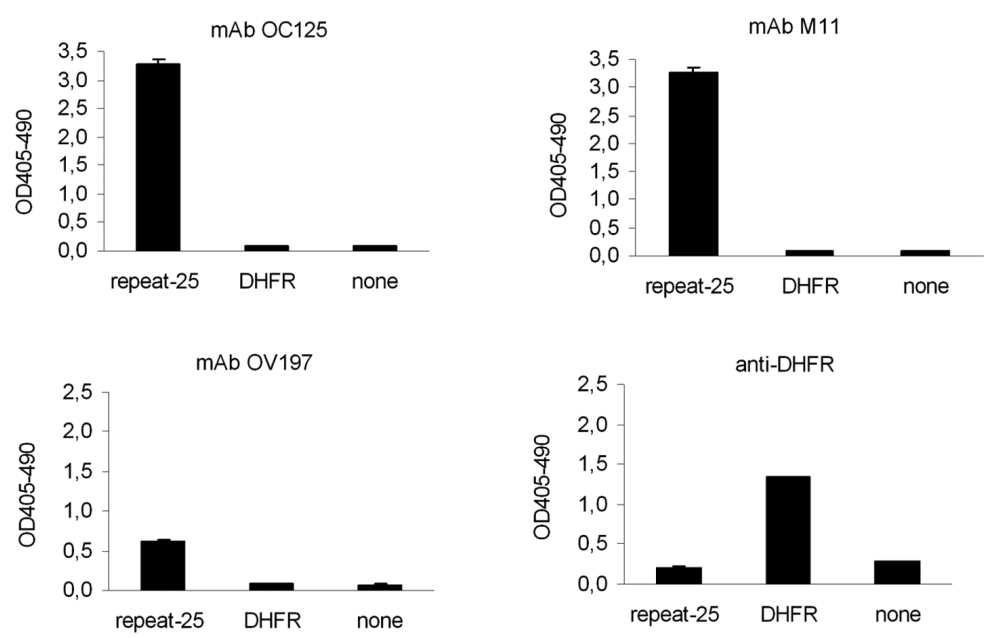

Fig. 5. ELISA analysis of R-25. E. coli extract containing the recombinant R-25 was tested with the OC125, M11, OV197 mAbs and with the polyclonal anti-murine DHFR as control antibody $(n=3)$. The recombinant murine DHFR was tested as mock protein.

the highest binding with R-51. The OV197 mAb bound R-25 and R-51 with high efficiency, it recognized also R-2 and R-9 and did not recognize the remaining repeats at all.

These data are in agreement with the differences observed in the amino acid sequences of CA125 repeats [34] and indicate that CA125 epitopes could be not homogeneously repeated in the tandem repeat region of the protein. This scenario is expected, considering that the repeated units are encoded by the evolving nucleotidic sequences of the tandem repeats, which may maintain or modify the epitope sequences after nucleotidic mutations. Besides, the alternative splicing of the numerous copies of the five exons encoding the repeat unit [34] may modulate the presence of epitopes. The greater number of repeats recognized by M11 mAb, respect to that recognized by OC125 and OV197 mAbs, indicates that the M11 epitope may be more represented in CA125 and could explain the different ability of these antibodies to bind the entire protein previously observed in immunoblots [14] and confirmed by our data (data not shown).

The molecular weights of the recombinant repeats observed in immunoblots were apparently not identical and often slightly different than those predicted from their amino acid sequences. The expression of the recombinant repeats in E. coli should rule out posttranslational modifications such as glycosilation [7]. If any proteolitic cleavage occurred in the bacterial environment, the recognition of all the repeats by the anti-histidine antibody and by the M11 mAb indicates that the region of the His-tagged SEA domain, necessary also for OC125 and OV197 binding, as shown by the deletion analysis of the R-25, has been preserved. Instead, the discrepancies among molecular weights could be due to the amino acid composition of these small fragments that could have influenced their electrophoretic run, as well as to a different negativecharge covering by SDS and/or by the poly-histidine sequence at the NH-terminal that may also retard the electrophoretic run of the recombinant repeats [21,28].

In order to map the epitopes of OC125, M11 and OV197 mAbs, a deletion analysis of R-25 amino acid sequence was performed through the subcloning and the expression in E. coli of partial regions of this repeat. The R-25 fragments were subcloned according to a scheme of overlapping regions from 50 up to 100 aa long, in order to maintain putative linear sequences recognized by the antibodies. The data, obtained with this deletion analysis, show that the shortest fragment recognized by anti-CA125 mAbs was an amino-terminal region 128 aa long, containing the entire SEA domain [24,31,32], indicating that the remaining 28 carboxy-terminal aa of the repeat do not participate to the formation of epitopes. This carboxyterminal region presents a turn structure downstream the last beta sheet of the SEA domain [32], and it may be heavily O-glycosilated [34] and not prone to interaction with antibodies.

Since continuous epitopes may have a size of a maximum of 15-22 aa [35], the necessity of the entire R25 SEA domain for the binding with OC125, M11 and OV197 mAbs, confirmed the conformational type of these epitopes and it was in agreement with the loss of M11 and OC125 epitopes after the R-11 digestion with endoprotease Lys-C or protease Asp-N [34] or with the 
pattern of the E. coli-proteolysed fragments observed in our immunoblots. Conformational epitopes, which could not be defined by deletion mapping or overlapping synthetic peptides, may be observed in Western blots after denaturing and reducing SDS-PAGE electrophoresis, due to their possible renaturation during the transfer on membrane and their maintenance in the subsequent steps of the method, as previously reported [30,39]. The signals observed in immunoblots, derived from the entire R-25 or from the 128 aa fragment, could be thereby due to the renatured conformation of the SEA domain. The epitope conformations are very probably destroyed in the shorter deleted fragments. We can not exclude that some of the R-25 regions, encoded by the delete constructs, may interact with antibodies in a native contest at least partially, and that their incorrect folding could be due to subcloning. Anyway, the data obtained with the chimeric DHFR/R25 fragments, i.e. using a different conformational context, were superimposable to data derived from notchimeric constructs, suggesting that the necessity of the entire R-25 SEA domain was independent from the subcloning strategy and that could be physiological.

The amino acid sequence within the R-11, known as "cysteine loop" (Fig. 1), has been reported as the site of interaction for the OC125 and M11 antibodies [34], on the basis of protease digestions of this recombinant repeat. These digestions resulted in the destruction of the epitopes without any direct evidence of antibody binding to this cysteine loop and the exact localization of the M11 and OC125 epitopes remained undetermined [7]. Nevertheless this region was synthesized as 21-mer fragment and still indicated as "peptide epitope" in further investigations [40]. Our data show that, although all the recombinant R-25 fragments encompassed the 21 aa region, they were not recognized by OC125, M11 and OV197 mAbs, neither in chimeric nor in not-chimeric constructs, with the exceptions of the fragment containing the SEA domain and the entire repeat. These data indicate that this 21 aa region is not sufficient for the interactions with the antibodies.

It is worth noting that the OV197 mAb recognized the recombinant R-25 better than the corresponding chimeric protein DHFR/R-25, and that in ELISA assays OV197 showed a lower binding-efficiency respect to OC125 and M11 antibodies. Since in chimeric constructs the NH-terminal of the R-25 is fused to the murine DHFR protein and in ELISA assays it is bound to the solid phase through the poly-histidine sequence, a steric-hindrance effect may be supposed and it might speculate that NH-terminal region of folded repeats could be in spatial proximity of OV197-epitope site.
The alignment of the amino acid sequences (Fig. 1) shows that, among the six recombinant repeats here reported, some substitutions could be responsible for their different immunological patterns; for instance, $\mathrm{K}$ 22, C-29, S-44 and V-93 are present only in R-25, which $\mathrm{N}$-terminal region of 128 amino acids was efficiently recognized by the antibodies from all the three CA125-mAb classes. Further investigation will be focused on the mutational analysis of the recombinant repeats, in order to identify those residues that might contribute to the conformational structure of CA125 epitopes.

Taking into consideration that other amino acid sequences for the repeats are present in CA125, in addition to those reported in this work, presented data suggest an epitope map for CA125 more complex than previously supposed. A diversified presence of CA125 epitopes along the tandem-repeat domain should be considered to find more effective immuno-therapies against the epithelial ovarian cancer, since it may reflect their availability as target antigens. Besides, repeats with an immunogenic profile not much represented could promote an immune response against CA125 also in human patients, thus representing good candidates for the vaccination against ovarian cancer.

\section{References}

[1] A.G. Zeimet, F.A. Offner, E. Müller-Holzner, M. Widschwendter, B. Abendstein, L.C. Fuith, G. Daxenbichler, C. Marth, Peritoneum and tissues of the female reproductive tract as physiological sources of CA-125, Tumour Biology 19 (1998), 275-282.

[2] A.P. Wilson, M. Dent, T. Pejovic, L. Hubbold, H. Radford, Characterisation of seven human ovarian tumour cell lines, British Journal of Cancer 74 (1996), 722-727.

[3] B.C. Schultes, R.P. Baum, A. Niesen, A.A. Noujaim, R. Madiyalakan, Anti-idiotype induction therapy: anti-CA125 antibodies (Ab3) mediated tumor killing in patients treated with Ovarex mAb B43.13 (Ab1), Cancer Immunology and Immunotherapy 46 (1998), 201-212.

[4] B.W.T. Yin, A. Dnistrian, K.O. Lloyd, Ovarian cancer antigen CA125 is encoded by the MUC16 mucin gene, International Journal of Cancer 98 (2002), 737-740.

[5] C. Thériault, M. Pinard, M. Comamala, M. Migneault, J. Beaudin, I. Matte, M. Boivin, A. Piché, C. Rancourt, MUC16 (CA125) regulates epithelial ovarian cancer cell growth, tumorigenesis and metastasis, Gynecologic Oncology 121 (2011), 434-443.

[6] D. Haridas, S. Chakraborty, M.P. Ponnusamy, I. Lakshmanan, S. Rachagani, E. Cruz, S. Kumar, S. Das, S.M. Lele, J.M. Anderson, U.A. Wittel, M.A. Hollingsworth, S.K. Batra, Pathobiological Implications of MUC16 Expression in Pancreatic Cancer, PLoS ONE 6 (2011), e26839. Epub 2011 Oct 31.

[7] D.J. Warren, K. Nustad, J.B. Beard, T.J. O'Brien, Expression and epitope characterization of a recombinant CA125 repeat: 
Fourth report from the ISOBM TD-1 Workshop, Tumour Biology 30 (2009), 51-60.

[8] H. Bouanene and a. Miled, Conflicting views on the molecular structure of the cancer antigen CA125/MUC16, Disease Markers 28 (2010), 385-394.

[9] H.M. Davis, V.R. Jr. Zurawski, R.C. Jr. Bast, T.L. Klug, Characterization of the CA 125 antigen associated with human epithelial ovarian carcinomas, Cancer Research 46 (1986), 6143-6148.

[10] H.W. de-Bruijn, C. van-Beeck-Calkoen, S. Jager, J.M. Duk, J.G. Aalders, G.J. Fleuren, The tumor marker CA 125 is a common constituent of normal cervical mucus, American Journal of Obstetrics and Gynecology 154 (1986), 10881091.

[11] I.K. Gipson, Human endocervical mucins, Ernst Schering Res. Found Workshop 52 (2005), 219-244.

[12] J. Pfister, P. Harter, C. Simonelli, M. Peters, J. Berek, P. Sabbatini, A. duBois, Abagovomab for ovarian cancer, Expert Opinion on Biological Therapy 11 (2011), 395-403.

[13] J.A.A. Gubbels, J. Belisle, M. Onda, C. Rancourt, M. Migneault, M. Ho, T.K. Bera, J. Connor, B.K. Sathynarayana, B. Lee, I. Pastan, M.S. Patankar, Mesothelin-MUC16 binding is a high affinity, $\mathrm{N}$-glycan dependent interaction that facilitates peritoneal metastasis of ovarian tumors, Molecular Cancer 5 (2006), 50-65.

[14] K. Nustad, R.C. Jr. Bast, T.J. O’Brien, O. Nilsson, P. Seguin, M.R. Suresh, T. Saga, S. Nozawa, O.P. Børmer, H.W. de Bruijn, M. Nap, A. Vitali, M. Gadnell, J. Clark, K. Shigemasa, B. Karlsson, F.T. Kreutz, D. Jette, H. Sakahara, K. Endo, E. Paus, D. Warren, S. Hammarström, P. Kenemans, J. Hilgers, Specificity and affinity of 26 monoclonal antibodies against the CA 125 antigen: first report from the ISOBM TD-1 workshop, International Society for Oncodevelopmental Biology and Medicine, Tumour Biology 17 (1996), 196-219.

[15] K. Nustad, Y. Lebedin, K.O. Lloyd, K. Shigemasa, H.W.A de Bruijn, B. Jansson, O. Nilsson, K.H. Olsen, T.J. O'Brien, Epitopes on CA 125 from cervical mucus and ascites fluid and characterization of six new antibodies, Tumour Biology 23 (2002), 303-314.

[16] K.O. Lloyd, B.W. Yin, Synthesis and secretion of the ovarian cancer antigen CA 125 by the human cancer cell line NIH: OVCAR-3, Tumour Biology 22 (2001), 77-82.

[17] K.O. Lloyd, B.W. Yin, V. Kudryashov, Isolation and characterization of ovarian cancer antigen CA 125 using a new monoclonal antibody (VK-8): identification as a mucin-type molecule, International Journal of Cancer 71(1997), 842850.

[18] L.F. Norum, B. Erikstein, K. Nustad, Elevated CA125 in breast cancer - A sign of advanced disease, Tumour Biology 22 (2001), 223-228.

[19] M. Binaschi, C. Simonelli, C. Goso, M. Bigioni, C.A. Maggi, Maintenance therapy in ovarian cancer: Molecular basis and therapeutic approach, Experimental and Therapeutic Medicine 2 (2011), 173-180.

[20] M.G. Muto, N.J. Finkler, A.I. Kassis, A.E. Howes, L.L. Anderson, C.C. Lau, V.R. Jr. Zurawski, K. Weadock, S.S. Tumeh, P. Lavin, R.C. Knapp, Intraperitoneal radioimmunotherapy of refractory ovarian carcinoma utilizing iodine-131-labeled monoclonal antibody OC125, Gynecologic Oncology 45 (1992), 265-272.

[21] M.J. Ward, H. Lew, D.R. Zusman, Social motility in Myxococcus xanthus requires FrzS, a protein with an extensive coiled-coil domain, Molecular Microbiology 37 (2000), 13571371.
[22] M.S. Patankar, Y. Jing, J.C. Morrison, J.A. Belisle, F.A. Lattanzio, Y. Deng, N.K. Wong, H.R. Morris, A. Dell, G.F. Clark, Potent suppression of natural killer cell response mediated by the ovarian tumor marker CA125, Gynecologic Oncology 99 (2005), 704-713.

[23] P. Argüeso, S. Spurr-Michaud, C.L. Russo, A. Tisdale, I.K. Gipson, MUC16 mucin is expressed by the human ocular surface epithelia and carries the H185 carbohydrate epitope, Investigative Ophthalmology and Visual Science 44 (2003), 2487-2495.

[24] P. Bork, L. Patthy, The SEA module: A new extracellular domain associated with O-glycosylation, Protein Science 4 (1995), 421-425.

[25] P.M. Fayers, G. Rustin, R. Wood, A. Nelstrop, R.C. Leonard, P. Wilkinson, D. Cruickshank, E.J. McAllister, C.W. Redman, D. Parker, I.V. Scott, M.L. Slevin, J.E. Roulston, The prognostic value of serum CA 125 in patients with advanced ovarian carcinoma: An analysis of 573 patients by the Medical Research Council Working Party on Gynecological Cancer, International Journal of Gynecological Cancer 3 (1993), 285292.

[26] R.C. Jr. Bast, F.J. Xu, Y.H. Yu, S. Barnhill, Z. G.B. Zhang, Mills, CA 125: The past and the future, The International Journal of Biological Markers 13 (1998), 179-187.

[27] R.C. Jr. Bast, M. Feeney, H. Lazarus, L.M. Nadler, R.B. Colvin, R.C. Knapp, Reactivity of a monoclonal antibody with human ovarian carcinoma, The Journal of Clinical Investigation 68 (1981), 1331-1337.

[28] S. Bar-Noy, J. Moskovitz, Mouse methionine sulfoxide reductase B: Effect of selenocysteine incorporation on its activity and expression of the seleno-containing enzyme in bacterial and mammalian cells, Biochemical and Biophysical Research Communications 297 (2002), 956-961.

[29] S. Reinartz, S. Failer, T. Schuell, U. Wagner, CA125 (MUC16) gene silencing suppresses growth properties of ovarian and breast cancer cells, European Journal of Cancer 48 (2011), 1558-1569.

[30] S.D. Dunn, Effects of the modification of transfer buffer composition and the renaturation of proteins in gels on the recognition of proteins on Western blots by monoclonal antibodies, Analytical Biochemistry 157 (1986), 144-153.

[31] S.J. Williams, D.H. Wreschner, M. Tran, H.J. Eyre, G.R. Sutherland, M.A. McGuckin, Muc13, a novel human cell surface mucin expressed by epithelial and hemopoietic cells, The Journal of Biological Chemistry 276 (2001), 18327-18336.

[32] T. Maeda, M. Inoue, S. Koshiba, T. Yabuki, M. Aoki, E. Nunokawa, E. Seki, T. Matsuda, Y. Motoda, A. Kobayashi, F. Hiroyasu, M. Shirouzu, T. Terada, N. Hayami, Y. Ishizuka, N. Shinya, A. Tatsuguchi, M. Yoshida, H. Hirota, Y. Matsuo, K. Tani, T. Arakawa, P. Carnici, J. Kawai, Y. Hayashizaki, T. Kigawa, S. Yokoyama, Solution structure of the SEA domain from the murine homologue of ovarian Cancer Antigen CA125 (MUC16), The Journal of Biological Chemistry 279 (2004), 13174-13182.

[33] T.J. O'Brien, J.B. Beard, L.J. Underwood, K. Shigemasa, The CA 125 gene: A newly discovered extension of the glycosylated $\mathrm{N}$-terminal domain doubles the size of this extracellular superstructure, Tumour Biology 23 (2002), 154-169.

[34] T.J. O'Brien, J.B. Beard, L.J. Underwood, R.A. Dennis, A.D. Santin, L. York, The CA 125 gene: An extracellular superstructure dominated by repeat sequences, Tumour Biology 22 (2001), 348-366

[35] W.G. Lawer, G.M. Air, R.G. Webster, S-J. Smith-Gill, Epi- 
topes on protein antigens: misconceptions and realities, Cell 61 (1990), 533-556.

[36] W.J. Murdoch, E.A. Van Kirk, A.M. Smedts, Complementinhibiting effect of ovarian cancer antigen CA-125, Cancer Letters 236 (2006), 54-57.

[37] Y. Chen, S. Clark, T. Wong, Y. Chen, Y. Chen, MS. Dennis, E. Luis, F. Zhong, S. Bheddah, H. Koeppen, A. Gogineni, S. Ross, P. Polakis, W. Mallet, Armed antibodies targeting the mucin repeats of the ovarian cancer antigen, MUC16, are highly efficacious in animal tumor models, Cancer Research 67 (2007), 4924-4932.

[38] Y. Matsuoka, K. Endo, Y. Kawamura, T. Yoshida, T. Saga,
Y. Watanabe, M. Koizumi, T. Nakashima, J. Konishi, N. Yamaguchi, R. Yatani, Normal bronchial mucus contains high levels of cancer-associated antigens, CA125, CA19-9, and carcinoembryonic antigen, Cancer 65 (1990), 506-510.

[39] Y-H. Zhou, Z. Chen, R.H. Purcell, S.U. Emerson, Positive reactions on western blots do not necessarily indicate the epitopes on antigens are continuous, Immunology and cell biology 85 (2007), 73-78.

[40] Z.T. Berman, L.J. Moore, K.E. Knudson, R.J. Whelan, Synthesis and structural characterization of the peptide epitope of the ovarian cancer biomarker CA125 (MUC16), Tumour Biology 31 (2010), 495-502. 


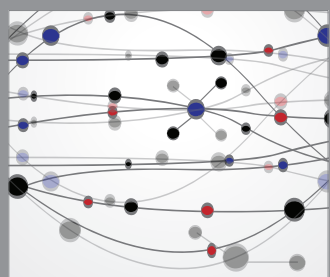

The Scientific World Journal
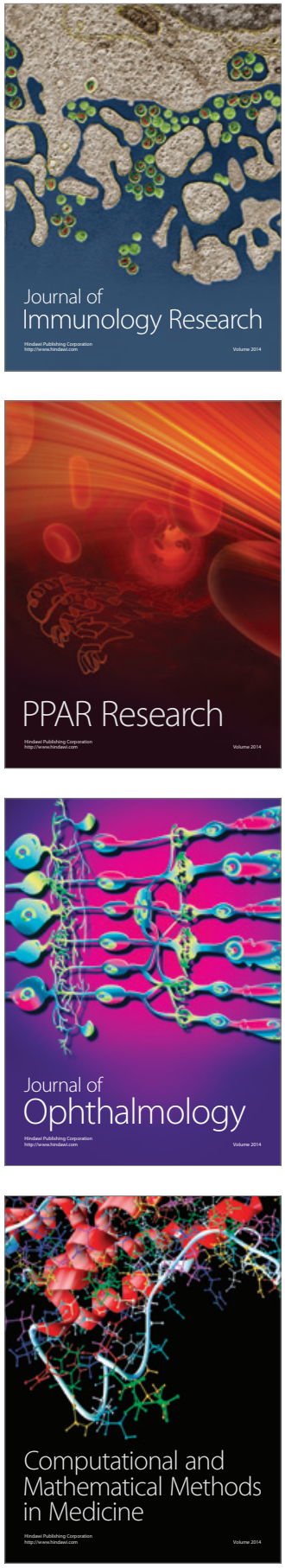

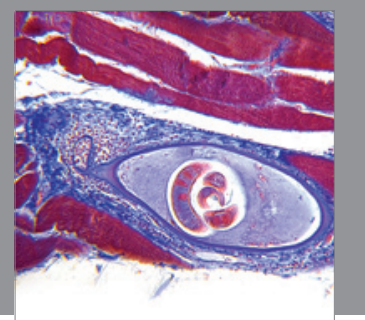

Gastroenterology

Research and Practice
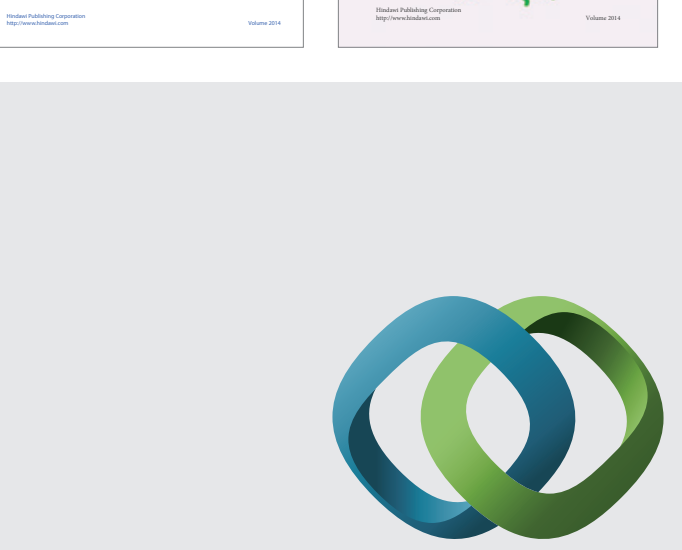

\section{Hindawi}

Submit your manuscripts at

http://www.hindawi.com
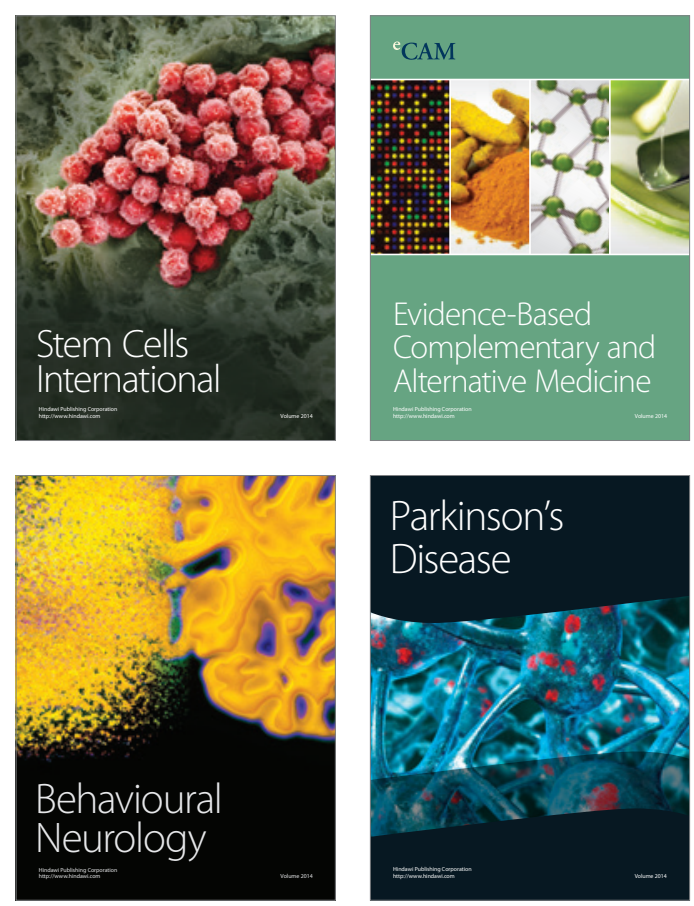

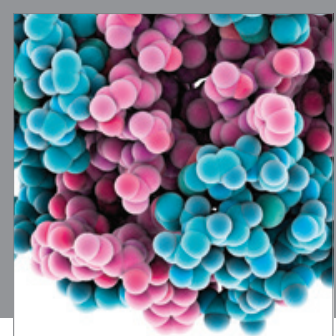

Journal of
Diabetes Research

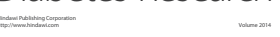

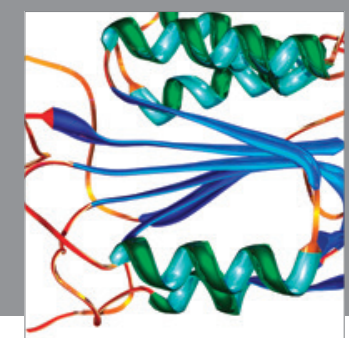

Disease Markers
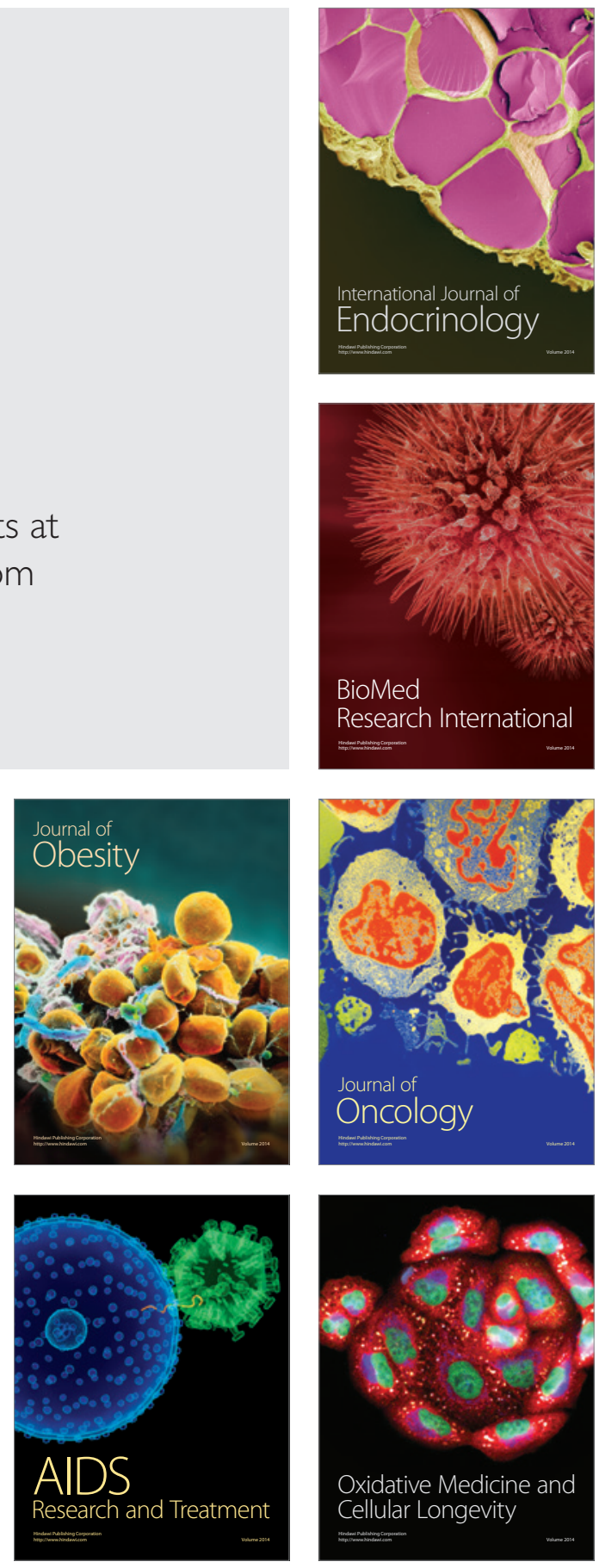